\title{
Comparison of FOLFIRINOX, Gemcitabine Plus Nab- Paclitaxel, and Chemoradiotherapy as First-Line Treatments for Locally Advanced Pancreatic Cancer
}

Shintaro Nakano ( $\nabla$ shintaro-n@frontier.hokudai.ac.jp )

Hokkaido University Hospital: Hokkaido Daigaku Byoin https://orcid.org/0000-0001-9630-572X

Yoshito Komatsu

Hokkaido University Hospital: Hokkaido Daigaku Byoin

\section{Yasuyuki Kawamoto}

Hokkaido University Hospital: Hokkaido Daigaku Byoin

\section{Rika Saito}

Hokkaido University Hospital: Hokkaido Daigaku Byoin

\section{Ken Ito}

Hokkaido University Hospital: Hokkaido Daigaku Byoin

Hiroshi Nakatsumi

Hokkaido University Hospital: Hokkaido Daigaku Byoin

\section{Kazuaki Harada}

Hokkaido University Hospital: Hokkaido Daigaku Byoin

\section{Satoshi Yuki}

Hokkaido University Hospital: Hokkaido Daigaku Byoin

Naoya Sakamoto

Hokkaido University Hospital: Hokkaido Daigaku Byoin

\section{Research Article}

Keywords: chemoradiotherapy, FOLFIRINOX, gemcitabine plus nab-paclitaxel, locally advanced pancreatic adenocarcinoma

Posted Date: March 30th, 2021

DOI: https://doi.org/10.21203/rs.3.rs-356113/v1

License: (c) (i) This work is licensed under a Creative Commons Attribution 4.0 International License. Read Full License 


\section{Abstract}

Background: Currently, it is unclear whether chemotherapy or chemoradiotherapy (CRT) is the optimal first-line treatment for patients with locally advanced pancreatic adenocarcinoma. In this study, we compared the efficacy and safety of FOLFIRINOX (FFX), gemcitabine plus nab-paclitaxel (GnP), and CRT as first-line treatments for locally advanced pancreatic adenocarcinoma.

Methods: We evaluated patients receiving FFX, GnP, or CRT, and assessed treatment efficacy in terms of overall survival and progression-free survival. Safety was evaluated using the Common Toxicity Criteria for Adverse Events (version 4.0).

Results: Fifty-five patients were included in the analysis (10 for FFX, 25 for GnP, and 20 for CRT). The median overall survival was 7.1, 16.9, and 20.0 months in the FFX, GnP, and CRT groups, respectively. There was no significant difference in overall survival between the FFX and GnP groups (HR: $0.503,95 \% \mathrm{Cl}: 0.205-1.238, p=0.135), \mathrm{FFX}$ and CRT groups (HR: $0.518,95 \% \mathrm{Cl}: 0.213-1.256, p=0.136)$, or GnP and CRT groups (HR: $0.993,95 \%$ Cl: $0.451-2.188, p=$ 0.987 ). The 1 -year survival rates were $40 \%, 64 \%$, and $60 \%$, whereas the 2 -year survival rates were $0 \%, 16 \%$, and $35 \%$ in the FFX, GnP, and CRT groups, respectively.

Conclusions: Both chemotherapy and CRT were effective and well tolerated. Thus, the combination of intensive chemotherapy and radiotherapy may be a beneficial treatment strategy.

\section{Introduction}

Pancreatic cancer is the seventh leading cause of cancer-related deaths worldwide[1]. In the metastatic stage, systemic intensive chemotherapies such as FOLFIRINOX (FFX)[2] and gemcitabine plus nab-paclitaxel (GnP)[3] significantly improve the overall survival (OS) of patients compared with gemcitabine monotherapy. However, intensive chemotherapy results in progression-free survival (PFS) of only approximately 6 months, and the 5-year survival rate is lower than $10 \%[2,3]$. Although it has been shown that GnP and FFX outperform conventional treatment with respect to improving the survival rate of patients with distant metastasis, it remains unclear which of the two treatments is superior because no randomized trials have compared the efficacy of GnP to that of FFX in patients with metastatic cancer. Furthermore, patients with locally advanced pancreatic cancer (LAPC) have been excluded from pivotal studies[2, 3], and a randomized phase II trial comparing these two regimens in patients with LAPC is still ongoing[4].

Meanwhile, chemoradiotherapy (CRT) has shown good outcomes[5-7]; thus, it is considered one of the standard treatment modalities for LAPC. In Japan, CRT combined with S-1, a combination of tegafur, gimeracil, and oteracil potassium, is recommended[5, 8, 9]. However, no randomized trial has compared the current standard combination chemotherapy and S-1-based CRT. Therefore, it remains unclear whether chemotherapy or CRT is the optimal first-line treatment modality for patients with LAPC. In this study, we compared the efficacy and safety of FFX, GnP, and CRT as first-line treatments for patients with LAPC.

\section{Results}

\section{Patient characteristics}

The clinicopathological characteristics of the patients are listed in Table 1. Overall, 55 patients were included in the analysis. Among them, 10, 25, and 20 patients received FFX, GnP, and CRT, respectively, as first-line treatments (Fig. 1). In the FFX group, six patients received modified FOLFIRINOX (mFFX) and four patients received FFX. In total, 10 
(100\%), 25 (100\%), and 19 (95\%) patients in the FFX, GnP, and CRT groups, respectively, had an Eastern Cooperative Oncology Group performance status of 0 or 1 . There were 10 (100\%), 24 (96\%), and 14 (70\%) patients in the FFX, GnP, and CRT groups, respectively, with pancreatic head cancer. Furthermore, one (10\%), six (24\%), and four (20\%) patients in the FFX, GnP, and CRT groups, respectively, had metastatic lymph nodes, and 6 (60\%), 9 (36\%), and 10 (50\%) patients, respectively, underwent stent implantation.

Table 1

Patient characteristics

\begin{tabular}{|c|c|c|c|c|c|}
\hline & & $\begin{array}{l}\text { FFX } \\
\text { group }\end{array}$ & GnP group & $\begin{array}{l}\text { CRT } \\
\text { group }\end{array}$ & \multirow[t]{2}{*}{$\begin{array}{l}p- \\
\text { value }\end{array}$} \\
\hline & & $N=10$ & $N=25$ & $N=20$ & \\
\hline Sex & Male/female & $7 / 3$ & $12 / 13$ & $13 / 7$ & $0.364^{*}$ \\
\hline \multirow[t]{2}{*}{ Age } & Median & 65.5 & 67 & 66 & \multirow[t]{2}{*}{$0.507^{+}$} \\
\hline & (range) & $(49-69)$ & $(33-72)$ & $(52-81)$ & \\
\hline PS & $0 / 1 / \geq 2$ & $6 / 4 / 0$ & $12 / 13 / 0$ & $6 / 13 / 1$ & $0.387^{\star}$ \\
\hline Tumor location & Head/body/tail & $10 / 0 / 0$ & $14 / 10 / 1$ & $14 / 6 / 0$ & $0.162^{*}$ \\
\hline Pathology & $\begin{array}{l}\text { Adenocarcinoma/adenosquamous } \\
\text { carcinoma }\end{array}$ & $9 / 1$ & $25 / 0$ & $20 / 0$ & $0.176^{\star}$ \\
\hline $\begin{array}{l}\text { Lymph node } \\
\text { metastasis }\end{array}$ & Yes/no & $1 / 9$ & $6 / 19$ & $4 / 16$ & $0.646^{\star}$ \\
\hline \multirow[t]{2}{*}{ CA19-9 } & Median & 112.5 & 120 & 206 & \multirow[t]{2}{*}{$0.800^{+}$} \\
\hline & (range) & $\begin{array}{l}(1.3- \\
1253)\end{array}$ & $\begin{array}{l}(1.0- \\
15586)\end{array}$ & $\begin{array}{l}(1- \\
7606)\end{array}$ & \\
\hline Stent & Yes/no & $6 / 4$ & $9 / 16$ & $10 / 10$ & $0.383^{*}$ \\
\hline \multicolumn{6}{|c|}{ *: Fisher's exact test } \\
\hline \multicolumn{6}{|c|}{ †: Kruskal-Wallis test } \\
\hline $\begin{array}{l}\text { FFX: FOLFIRIN } \\
\text { - 9: colorectal }\end{array}$ & Semcitabine plus nab-paclitaxel, $\mathrm{Cl}$ & radioth & & ance sta & A 19 \\
\hline
\end{tabular}

\section{Treatment Exposure}

On 30 September 2019, the cut-off date, 51 patients discontinued first-line treatment with a median follow-up time of 13.3 months. In total, six (60\%), eight (32\%), and two (10\%) patients in the FFX, GnP, and CRT groups, respectively, discontinued treatment because of disease progression. Meanwhile, 7 (70\%), 16 (64\%), and 18 (90\%) patients in the FFX, GnP, and CRT groups, respectively, received the subsequent therapy (Table 2). In the FFX group, three (30\%) patients received gemcitabine monotherapy or GnP. In the GnP group, eight (32\%) patients received CRT, and three (12\%) patients underwent conversion surgery. In the CRT group, 14 (70\%) patients received GnP, whereas 3 (15\%) patients received gemcitabine monotherapy. In the FFX group, the median relative dose intensities of bolus 5-FU, civ 5$\mathrm{FU}$, irinotecan, and oxaliplatin were $0.12,0.82,0.72$, and 0.77 , respectively. In the mFFX group, the median relative dose intensities of civ $5-\mathrm{FU}$, irinotecan, and oxaliplatin were $0.7,0.56$, and 0.48 , respectively. The median relative dose 
intensity of irinotecan was 0.72 in the FFX group and 0.55 in the mFFX group. In the GnP group, the median relative dose intensities of gemcitabine and nab-paclitaxel were 0.76 and 0.68 , respectively.

Table 2

Post-treatment outcomes

\begin{tabular}{|c|c|c|c|c|}
\hline & & $\begin{array}{l}\text { FFX group } \\
N=10\end{array}$ & $\begin{array}{l}\text { GnP group } \\
N=25\end{array}$ & $\begin{array}{l}\text { CRT group } \\
N=20\end{array}$ \\
\hline Discontinued treatment & & 10 & 21 & \\
\hline \multirow[t]{6}{*}{ Reason } & Progression & 6 & 8 & 3 \\
\hline & Adverse events & 2 & 3 & 0 \\
\hline & Conversion surgery & 0 & 3 & 0 \\
\hline & Completion of CRT & - & - & 17 \\
\hline & Change to CRT & 1 & 7 & - \\
\hline & Others & 1 & 0 & 0 \\
\hline Post-treatment & Yes/no & $7 / 2$ & $16 / 5$ & $18 / 2$ \\
\hline Conversion surgery & FFX & 0 & 3 & 0 \\
\hline \multirow[t]{6}{*}{ Chemotherapy } & FOLFOX & - & 1 & 0 \\
\hline & GnP & - & 1 & 0 \\
\hline & GEM & 3 & - & 14 \\
\hline & CRT & 3 & 1 & 3 \\
\hline & S-1 & 0 & 8 & - \\
\hline & & 1 & 3 & 1 \\
\hline
\end{tabular}

\section{Efficacy}

The treatment responses of the mFFX and GnP groups are summarized in Table 3. Of the 55 patients, 49 patients had measurable lesions. The objective response rates were $11 \%, 17 \%$, and $6 \%$, whereas the disease control rates were $67 \%$, $78 \%$, and 77\% in the FFX, GnP, and CRT groups, respectively. The median PFS was 4.2 months (95\% Cl: $3.1-5.2)$ in the FFX group and 3.9 months (95\% Cl: 2.9-4.8) in the GnP group (HR: 1.033, 95\% Cl: 0.465-2.299) (Fig. 2a), with no significant difference (HR: $1.033,95 \% \mathrm{Cl}: 0.465-2.299, p=0.936)$. The median PFS of the CRT group was defined from the start of CRT to the first progression, because almost all patients in this group discontinued treatment at the end of radiotherapy and changed regimens. The median PFS of the CRT group was 5.6 months (95\% CI: 2.1-9.1).

The median OS of the FFX, GnP, and CRT groups was 7.1 months (95\% Cl: 2.7-11.6), 16.9 months (95\% Cl: 11.7-22.2), and 20.0 months (95\% Cl: 8.9-31.1), respectively. There was no significant difference in OS between the FFX and GnP (HR: $0.503,95 \% \mathrm{Cl}, 0.205-1.238, p=0.135)$, FFX and CRT (HR: 0.518, 95\% Cl, 0.213-1.256, $p=0.146$ ), and GnP and CRT (HR: 0.993, 95\% Cl: 0.451-2.188, $p=0.987$ ) groups (Fig. 2b). The 1-year survival rates were 40\%, 64\%, and 60\%, whereas the 2 -year survival rates were $0 \%, 16 \%$, and $35 \%$, in the FFX, GnP, and CRT groups, respectively. 
Table 3

Treatment response

\begin{tabular}{|lcccccccc|}
\hline & N & CR & PR & SD & PD & NE & RR (\%) & DCR (\%) \\
\hline FFX group & 9 & 0 & 1 & 5 & 2 & 1 & 11 & 67 \\
\hline GnP group & 23 & 0 & 4 & 14 & 2 & 3 & 17 & 78 \\
\hline CRT group & 17 & 0 & 1 & 12 & 3 & 1 & 6 & 77 \\
\hline
\end{tabular}

$\mathrm{RR}: p=0.734^{*}$

DCR: $p=0.825^{\star}$

* Fisher's exact test

FFX: FOLFIRINOX, GnP: Gemcitabine plus nab-paclitaxel, CRT: chemoradiotherapy, CR: complete response, PR: partial response, SD: stable disease, PD: progressive disease, NE: not evaluable, RR: response rate, DCR: disease control rate, N: number of patients.

\section{Safety Analysis}

The treatment-related adverse events (AEs) are shown in Table 4. No patient died of a treatment-related cause. Among the hematological AEs, severe (grade 3 or 4 ) neutropenia was significantly more frequent in the FFX (70\%) and GnP $(44 \%)$ groups than in the CRT group $(0 \%)(p<0.001)$, whereas nausea was more frequent in the CRT group (70\%) than in the chemotherapy groups ( $32 \%$ in the FFX group and $40 \%$ in the GnP group). 
Table 4

Safety profile

\begin{tabular}{|lllllll|}
\hline & \multicolumn{1}{l}{ FFX group } & \multicolumn{2}{l|}{ GnP group } & \multicolumn{2}{l|}{ CRT group } \\
& N=10 & N=25 & \multicolumn{2}{c|}{$\mathbf{N = 2 0}$} & \\
\hline & All & $\geq$ G3 & All & $\geq$ G3 & All & $\geq$ G3 \\
\hline Leukopenia & $8(80)$ & $3(30)$ & $16(64)$ & $6(24)$ & $8(40)$ & $1(5)$ \\
\hline Neutropenia & $8(80)$ & $7(70)$ & $18(72)$ & $11(44)$ & $7(35)$ & 0 \\
\hline Anemia & $9(90)$ & $1(10)$ & $24(96)$ & $3(12)$ & $14(70)$ & $1(5)$ \\
\hline Thrombocytopenia & $9(90)$ & 0 & $15(60)$ & 0 & $12(60)$ & 0 \\
\hline Febrile neutropenia & $1(10)$ & $1(10)$ & 0 & 0 & 0 & 0 \\
\hline Nausea & $4(40)$ & 0 & $8(32)$ & 0 & $14(70)$ & 0 \\
\hline Vomiting & 0 & 0 & $1(4)$ & 0 & $2(10)$ & 0 \\
\hline Fatigue & $5(50)$ & $1(10)$ & $12(48)$ & 0 & $5(25)$ & 0 \\
\hline Anorexia & $8(80)$ & $1(10)$ & $13(52)$ & 0 & $14(70)$ & 0 \\
\hline Diarrhea & $5(50)$ & 0 & $4(16)$ & 0 & $4(20)$ & 0 \\
\hline Alopecia & $3(30)$ & - & $12(48)$ & - & 0 & - \\
\hline Peripheral sensory neuropathy & $4(40)$ & $1(4)$ & $14(56)$ & $1(4)$ & 0 & 0 \\
\hline FFX: FOLFIRINOX, GnP: Gemcitabine plus nab-paclitaxel, CRT: chemoradiotherapy. & \\
\hline
\end{tabular}

\section{Discussion}

In this study, we compared the efficacy and safety of FFX, GnP, and CRT as first-line treatments for LAPC. We found that both chemotherapy and CRT were effective for LAPC. In general, patients with LAPC have better outcomes than those with metastatic pancreatic cancer $[10,11]$. Thus, the treatment strategy differs between them. Conversion surgery is sometimes considered for LAPC, whereas surgery is not recommended for metastatic pancreatic cancer $[9,12]$. Currently, metastatic pancreatic cancer and LAPC are regarded as different entities, and patients have been separately enrolled for different trials. Among patients with LAPC, borderline resectable pancreatic cancer is associated with better outcomes than truly unresectable tumors. Therefore, we excluded patients with borderline resectable pancreatic cancer from the present study. As for efficacy, the FFX group showed similar PFS to the GnP group, but the median survival time of the FFX group was shorter than that of the GnP and CRT groups. The difference in post first-line treatment time between the FFX and GnP groups contributed to the difference in median survival time between these groups. In the FFX group, almost half of the patients received gemcitabine monotherapy as second-line treatment. This was less effective than second-line treatment, which included CRT and conversion surgery, in the GnP group. We did not conclude that FFX was less effective than GnP for patients with LAPC because of the small number of patients and the retrospective design of the present study.

Evidence of the efficacy and safety of FFX and GnP for LAPC is limited. In a multicenter observational study in Japan, FFX showed good OS of 18.5 months for LAPC[13]. GnP for unresectable LAPC also showed good PFS of 9.4 months and OS of 19.6 months in a multicenter retrospective study[14]. However, evidence from randomized studies of the efficacy of FFX and GnP for LAPC is lacking. Nevertheless, considering the improved clinical outcomes for patients 
with metastatic pancreatic cancer[2, 3] and given the supporting conclusions from observational and retrospective studies[13, 14], intensive chemotherapies appear to have clinical benefits in LAPC.

CRT is considered to be effective for local control, but not for preventing distant metastasis[9]. Therefore, using CRT confers a risk of early progression and distant metastasis. In this study, we found that first-line CRT was well tolerated and enabled good disease control. No patient in the CRT group developed progressive disease with distant metastasis at the end of the treatment.

The optimal treatment modality (CRT vs. chemotherapy) for patients with LAPC remains unclear. Several randomized trials have shown a clinical benefit of CRT for patients with LAPC. The combination of 5-FU and CRT (50.4 Gy) has shown better OS than the best supportive care[6]. The combination of gemcitabine and CRT has also resulted in better survival than gemcitabine monotherapy[7]. However, the chemotherapy regimen used in previous trials is less effective than the current standard regimen of FFX or GnP. There has been no randomized controlled trial comparing CRT and the current standard intensive regimen (FFX or GnP). In addition, there has been no randomized controlled trial comparing chemotherapy with the combination of S-1 and CRT, which has shown good outcomes for patients with LAPC and is the current standard regimen in Japan[5]. Furthermore, the maintenance therapy of previous clinical trials of S-1 with concurrent radiation therapy was S-1 monotherapy[5] or gemcitabine monotherapy[15], which may be insufficient. The present study shows that chemotherapy and CRT have similar efficacy. CRT has been reported to be fairly effective in preventing early metastasis. In this study, none of the patients in the CRT group experienced early metastasis; however, one patient in the CRT group developed early disease progression in the primary site. Many patients received GnP as maintenance therapy after CRT, which may have contributed to the good outcomes of CRT. As for safety, chemotherapy and CRT showed different trends in AEs. Hematological AEs such as neutropenia were more frequent in the chemotherapy groups than in the CRT group, whereas nausea was more common in the CRT group than in the chemotherapy groups. Considering the difference in safety profile may help in selecting a treatment.

Biomarkers are also important in selecting a treatment. Several studies have attempted to develop a classification based on tumor molecular profiles[16-20]. Moffitt et al. classified pancreatic cancer into two types, classical and basal-like[20]. The classical type has a better median OS than the basal-like type. Notably, FFX had worse efficacy in the basal-like type than in the classical type, whereas GnP showed similar efficacy in both[21]. This classification may be helpful in selecting the appropriate chemotherapy regimen. As for GnP, the activity of cytidine deaminase, which converts gemcitabine to its inactive form, is associated with the efficacy and toxicity of gemcitabine-containing chemotherapy[22, 23].

The present study has some limitations. First, this is a single-center retrospective study and included a relatively small number of patients. Second, the treatment strategies were chosen by the patients, which might have introduced selection bias. Prospective trials comparing chemotherapy and CRT are needed to verify our findings.

In conclusion, both chemotherapy and CRT were well tolerated and showed similar efficacy for LAPC. CRT as first-line treatment did not increase the risk of early disease progression and distant metastasis. Thus, the combination of intensive chemotherapy and CRT may be a good strategy for LAPC.

\section{Methods}

\section{Patients and study design}

This was a retrospective study of patients with LAPC who received FFX, GnP, or S-1 with concurrent radiation therapy as first-line treatment between January 2014 and December 2018 at Hokkaido University Hospital. The eligibility 
criteria were patients $\geq 20$ years of age, with histologically or cytologically confirmed pancreatic adenocarcinoma. This study was approved by the institutional review board of Hokkaido University Hospital (approval number: 019-0074). The need for informed consent was waived because of the retrospective nature of the study. This research was announced on a website (http://www.huhp.hokudai.ac.jp/).

\section{Treatment Protocol}

All treatment protocols were continued until disease progression, occurrence of unacceptable AEs, or patient's refusal to continue. All treatment plans were prepared by the attending physicians, and the patients selected their preferred treatment.

\section{FOLFIRINOX}

This regimen was administered as follows: 2-h intravenous (i.v.) infusion of $85 \mathrm{mg} / \mathrm{m}^{2}$ oxaliplatin and 2-h i.v. infusion of $200 \mathrm{mg} / \mathrm{m}^{2}$ l-leucovorin (during which $180 \mathrm{mg} / \mathrm{m}^{2}$ irinotecan was also i.v. infused for over $90 \mathrm{~min}$ ), followed by an i.v. bolus of $400 \mathrm{mg} / \mathrm{m}^{2} 5$-FU and continuous i.v. infusion of $2400 \mathrm{mg} / \mathrm{m}^{2} 5$-FU for over $46 \mathrm{~h}[2]$.

\section{Modified FFX}

mFFX was administered as follows: 2-h i.v. infusion of $85 \mathrm{mg} / \mathrm{m}^{2}$ oxaliplatin and 2-h i.v. infusion of $200 \mathrm{mg} / \mathrm{m}^{2} \mathrm{I}-$ leucovorin. Irinotecan $\left(150 \mathrm{mg} / \mathrm{m}^{2}\right)$ was also i.v. infused for over $90 \mathrm{~min}$, followed by continuous i.v. infusion of 2400 $\mathrm{mg} / \mathrm{m}^{2}$ 5-FU for over $46 \mathrm{~h}$ every 2 weeks. Bolus 5-FU was omitted[24].

\section{Gemcitabine plus nab-paclitaxel}

This regimen was administered as follows: 30-min i.v. infusion of $125 \mathrm{mg} / \mathrm{m}^{2}$ nab-paclitaxel, followed by 30 -min i.v. infusion of $1000 \mathrm{mg} / \mathrm{m}^{2}$ gemcitabine on days 1, 8, and 15 in a 4-week cycle[3].

\section{Chemoradiotherapy}

S-1 was administered orally at a dose of $40 \mathrm{mg} / \mathrm{m}^{2}$ twice on the day of irradiation during radiation therapy. The initial doses were determined according to the body surface area (BSA) as follows: patients with a BSA of $<1.25 \mathrm{~m}^{2}$ received $40 \mathrm{mg} /$ dose, BSA of $1.25-1.5 \mathrm{~m}^{2}$ received $50 \mathrm{mg} /$ dose, and BSA of $\geq 1.5 \mathrm{~m}^{2}$ received $60 \mathrm{mg} /$ dose[5]. Radiotherapy was initiated on day 1 of the treatment period using 10-MV photons. A fractional daily dose of 1.8 Gy (5 days/week) at the isocenter, up to a total dose of $50.4 \mathrm{~Gy}$, was prescribed[5].

\section{Outcome Assessment}

Outcome assessment

Efficacy was assessed using OS, which was measured from the date of treatment initiation to the date of death or the date of the last follow-up, and PFS. PFS was defined as the time from the date of treatment initiation to the first documentation of disease progression or death. Patients whose treatment regimens were changed without evidence of progression were censored. Tumor response was evaluated according to the Response Evaluation Criteria in Solid Tumors (v. 1.1)[25]. Toxicity was graded following the Common Toxicity Criteria for Adverse Events (v. 4.0)[26].

\section{Statistical analysis}


Qualitative and quantitative variables were compared using the chi-square test or Fisher's test and using a nonparametric (Wilcoxon) test, respectively. Data are presented with 95\% confidence intervals calculated using standard methods based on a binomial distribution. Survival was analyzed using the Kaplan-Meier method and compared according to treatment regimens (FFX, GnP, and CRT) using a log-rank test and the Cox proportional hazard model. Both efficacy and safety analyses included all patients who received at least one dose of chemotherapy. All analyses were conducted using SPSS v. 25 (IBM, Armonk, NY, USA). All tests were two-sided, and results with $p$-values of $<0.05$ were considered statistically significant.

\section{Abbreviations}

AE, adverse event; CRT, chemoradiotherapy; FFX, FOLFIRINOX; GnP, gemcitabine plus nab-paclitaxel; LAPC, Iocally advanced pancreatic cancer; OS, overall survival; PFS, progression-free survival.

\section{Declarations}

Ethics approval and consent to participate: All procedures were conducted in accordance with the ethical standards of the responsible committees on human experimentation (institutional and national) and with the Helsinki Declaration of 1964 and later versions. This study was approved by the institutional review board of Hokkaido University Hospital (approval number: 019-0074). The need for informed consent was waived owing to the retrospective nature of the study.

\section{Consent for publication}

The need for informed consent was waived owing to the retrospective nature of the study.

\section{Availability of data and materials}

The datasets generated and/or analyzed during the current study are available from the corresponding author on reasonable request.

\section{Competing interests}

Yoshito Komatsu received honoraria and research grants from Taiho, Yakult, and Daichi-Sankyo. The remaining authors declare no potential conflict of interest.

\section{Funding}

Not applicable.

\section{Authors' contributions}

All authors contributed to the study conception and design. Material preparation, data collection, and analysis were performed by S.N., Yoshito Komatsu., and Yasuyuki Kawamoto. The first draft of the manuscript was written by S.N., and all authors commented on previous versions of the manuscript. All authors read and approved the final manuscript.

\section{Acknowledgements}

We thank the patients and their families for participating in the study. We would like to thank Editage (www.editage.com) for English language editing. 


\section{References}

1. International Agency for Research on Cancer. WHO Global Cancer Observatory. 2018. https://gco.iarc.fr/. Accessed 11 May 2020.

2. Conroy T, Desseigne F, Ychou M, Bouché O, Guimbaud R, Bécouarn Y, et al. FOLFIRINOX versus gemcitabine for metastatic pancreatic cancer. N Engl J Med. 2011. doi:10.1056/nejmoa1011923.

3. Von Hoff DD, Ervin T, Arena FP, Chiorean EG, Infante J, Moore M, et al. Increased survival in pancreatic cancer with nab-paclitaxel plus gemcitabine. N Engl J Med. 2013. doi:10.1056/nejmoa1304369.

4. Mizusawa J, Fukutomi A, Katayama H, Ishii H, loka T, Okusaka T, et al. Protocol digest of randomized phase II study of modified FOLFIRINOX versus gemcitabine plus nab-paclitaxel combination therapy for locally advanced pancreatic cancer: Japan clinical oncology group study (JCOG1407). Pancreatology. 2018. doi:10.1016/j.pan.2018.07.007.

5. Ikeda M, loka T, Ito Y, Yonemoto N, Nagase M, Yamao K, et al. A multicenter phase II trial of S-1 with concurrent radiation therapy for locally advanced pancreatic cancer. Int J Radiat Oncol Biol Phys. 2013. doi:10.1016/j.jijrobp.2012.03.059.

6. Shinchi H, Takao S, Noma H, Matsuo Y, Mataki Y, Mori S, et al. Length and quality of survival after external-beam radiotherapy with concurrent continuous 5 -fluorouracil infusion for locally unresectable pancreatic cancer. Int $\mathrm{J}$ Radiat Oncol Biol Phys. 2002. doi:10.1016/s0360-3016(01)02806-1.

7. Loehrer PJ, Sr, Feng Y, Cardenes H, Wagner L, Brell JM, Cella D, et al. Gemcitabine alone versus gemcitabine plus radiotherapy in patients with locally advanced pancreatic cancer: an Eastern Cooperative Oncology Group trial. J Clin Oncol. 2011. doi:10.1200/jco.2011.34.8904.

8. loka T, Komatsu Y, Mizuno N, Tsuji A, Ohkawa S, Tanaka M, et al. Randomised phase II trial of irinotecan plus S-1 in patients with gemcitabine-refractory pancreatic cancer. Br J Cancer. 2017. doi:10.1038/bjc.2016.436.

9. Society JP. Clinical Practice Guidelines for Pancreatic Cancer 2019. Tokyo: Kanehara-shuppan; 2019.

10. Ueno H, loka T, Ikeda M, Ohkawa S, Yanagimoto H, Boku N, et al. Randomized phase III study of gemcitabine plus S-1, S-1 alone, or gemcitabine alone in patients with locally advanced and metastatic pancreatic cancer in Japan and Taiwan: GEST study. J Clin Oncol. 2013. doi:10.1200/jco.2012.43.3680.

11. Ishii H, Furuse J, Boku N, Okusaka T, Ikeda M, Ohkawa S, et al. Phase II study of gemcitabine chemotherapy alone for locally advanced pancreatic carcinoma: JCOG0506. Jpn J Clin Oncol. 2010. doi:10.1093/jjco/hyq011.

12. Network. NCC Gastric Cancer (Version 3). 2019.

13. Matsumoto I, Kamei K, Omae K, Suzuki S, Matsuoka H, Mizuno N, et al. FOLFIRINOX for locally advanced pancreatic cancer: Results and prognostic factors of subset analysis from a nation-wide multicenter observational study in Japan. Pancreatology. 2019. doi:10.1016/j.pan.2019.01.001.

14. Tanimoto A, Yagisawa M, Nakano S, Kawamoto Y, Yuki S, Ishiguro A, et al. 195P - HGCSG1601: a retrospective cohort study of the efficacy and safety of nab-paclitaxel plus gemcitabine (nab-P + GEM) for unresectable locally advanced pancreatic cancer (LAPC). Ann Oncol. 2019. doi:10.1093/annonc/mdz422.071.

15. loka T, Fukutomi A, Mizusawa J, Katayama H, Nakamura S, Ito Y, et al. Randomized phase II study of S-1 and concurrent radiotherapy with versus without induction chemotherapy of gemcitabine for locally advanced pancreatic cancer (LAPC): final analysis of JCOG1106. Ann Oncol. 2016. doi:10.1093/annonc/mdw371.13.

16. Connor AA, Denroche RE, Jang GH, Timms L, Kalimuthu SN, Selander I, et al. Association of distinct mutational signatures with correlates of increased immune activity in pancreatic ductal adenocarcinoma. JAMA Oncol. 2017. doi:10.1001/jamaoncol.2016.3916. 
17. Waddell N, Pajic M, Patch AM, Chang DK, Kassahn KS, Bailey P, et al. Whole genomes redefine the mutational landscape of pancreatic cancer. Nature. 2015. doi:10.1038/nature14169.

18. Collisson EA, Sadanandam A, Olson P, Gibb WJ, Truitt M, Gu S, et al. Subtypes of pancreatic ductal adenocarcinoma and their differing responses to therapy. Nat Med. 2011. doi:10.1038/nm.2344.

19. Bailey P, Chang DK, Nones K, Johns AL, Patch AM, Gingras MC, et al. Genomic analyses identify molecular subtypes of pancreatic cancer. Nature. 2016. doi:10.1038/nature16965.

20. Moffitt RA, Marayati R, Flate EL, Volmar KE, Loeza SG, Hoadley KA, et al. Virtual microdissection identifies distinct tumor- and stroma-specific subtypes of pancreatic ductal adenocarcinoma. Nat Genet. 2015. doi:10.1038/ng.3398.

21. O'Kane GM, Fischer S, Denroche R, Jang GH, Zhang A, Dodd A, et al. Integrative molecular profiling and response to chemotherapy on the COMPASS trial. J Clin Oncol. 2019. doi:10.1200/JC0.2019.37.4_suppl.188.

22. Cohen R, Preta LH, Joste V, Curis E, Huillard O, Jouinot A, et al. Determinants of the interindividual variability in serum cytidine deaminase activity of patients with solid tumours. Br J Clin Pharmacol. 2019. doi:10.1111/bcp.13849.

23. Tibaldi C, Camerini A, Tiseo M, Mazzoni F, Barbieri F, Vittimberga I, et al. Cytidine deaminase enzymatic activity is a prognostic biomarker in gemcitabine/platinum-treated advanced non-small-cell lung cancer: a prospective validation study. Br J Cancer. 2018. doi:10.1038/s41416-018-0307-3.

24. Ozaka M, Ishii H, Sato T, Ueno M, Ikeda M, Uesugi K, et al. A phase II study of modified FOLFIRINOX for chemotherapy-naive patients with metastatic pancreatic cancer. Cancer Chemother Pharmacol. 2018. doi:10.1007/s00280-018-3577-9.

25. Eisenhauer EA, Therasse P, Bogaerts J, Schwartz LH, Sargent D, Ford R, et al. New response evaluation criteria in solid tumours: revised RECIST guideline (version 1.1). Eur J Cancer. 2009. doi:10.1016/j.ejca.2008.10.026.

26. Common Terminology Criteria for Adverse Events v5.0 (CTCAE). 2017. https://ctep.cancer.gov/protocoldevelopment/electronic_applications/docs/CTCAE_v5_Quick_Reference_5x7.pdf/. Accessed 11 May 2020.

\section{Figures}

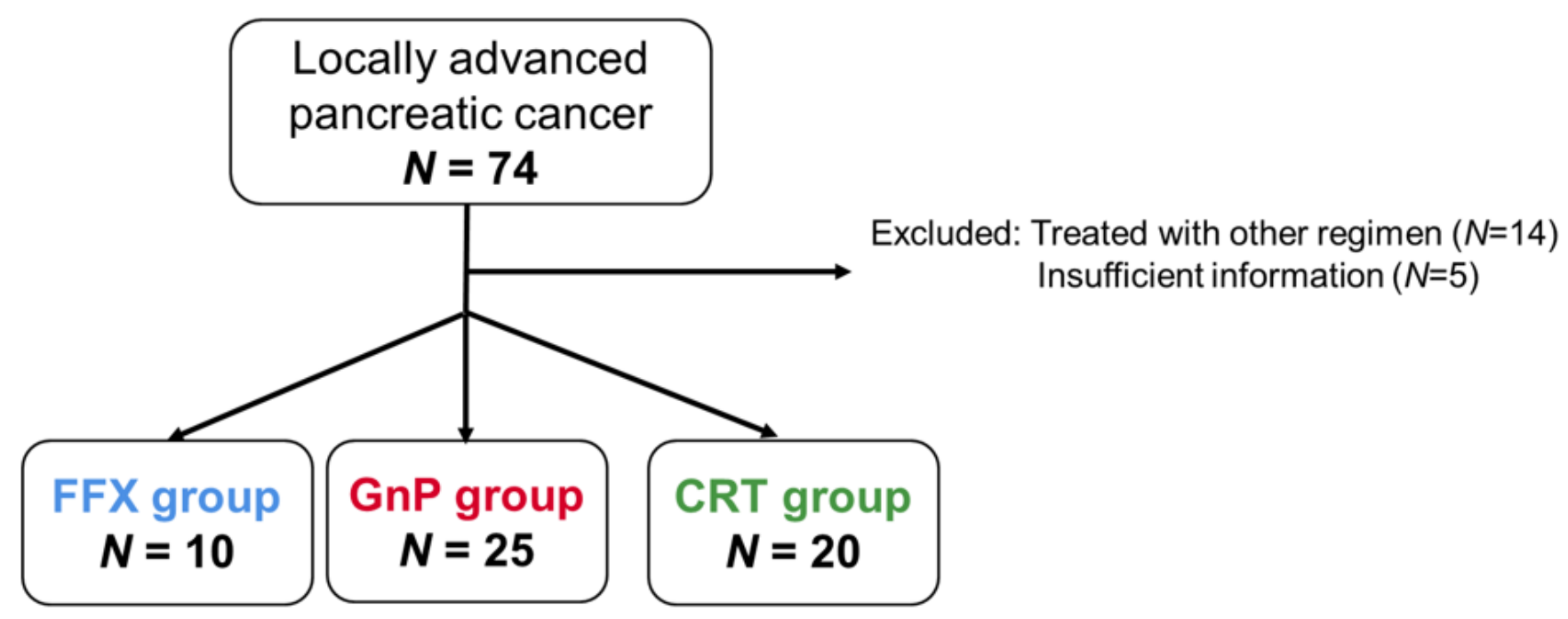

Figure 1

Page $11 / 12$ 
Patient inclusion flowchart. FFX: FOLFIRINOX, GnP: Gemcitabine plus nab-paclitaxel, CRT: chemoradiotherapy.

(a)

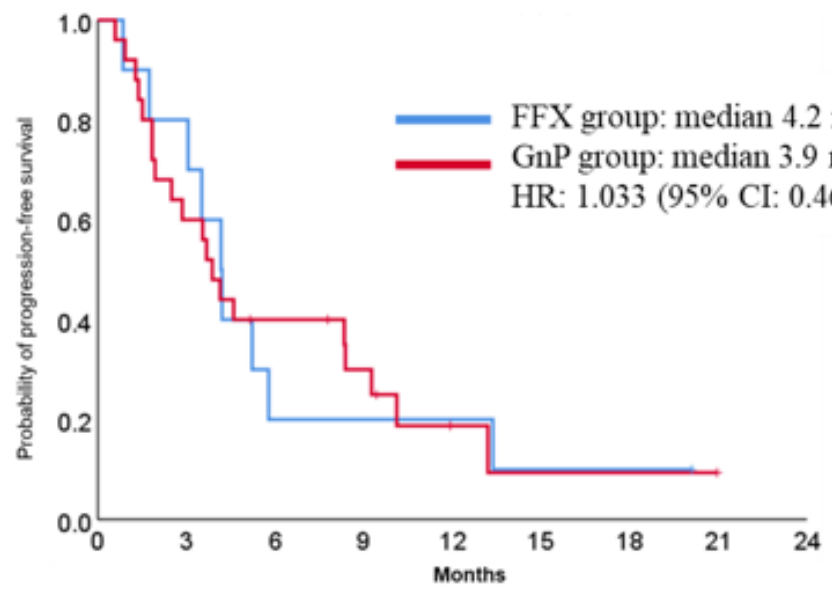

(b)

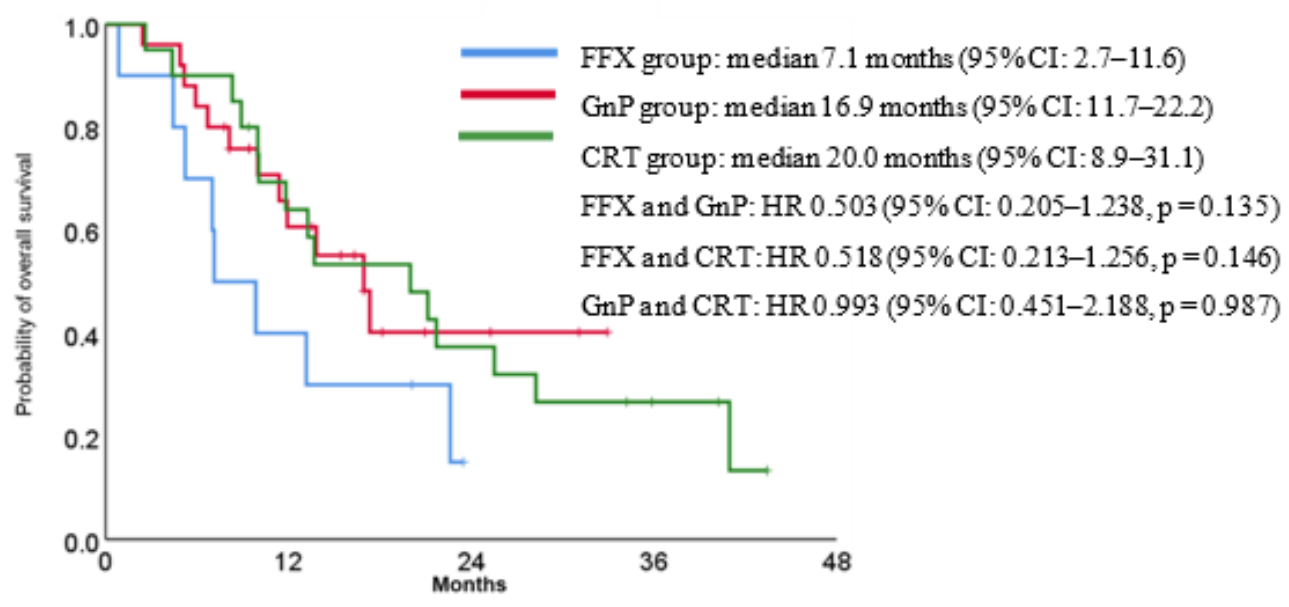

Figure 2

Kaplan-Meier curves for (a) progression-free survival and (b) overall survival. FFX: FOLFIRINOX, GnP: Gemcitabine plus nab-paclitaxel, CRT: chemoradiotherapy. 